\title{
Zum Verhältnis von Einzelhandel und Raumplanung in der Schweiz
}

\section{Der Einzelhandel im Planungsprozeß}

Das Verhältnis von Raumplanung und Stadtentwicklung zum Einzelhandel ist ein sehr pragmatisches: es gibt kaum übergeordnete Strategieziele der Raumplanung zu Fragen der Güterversorgung oder entsprechende Konzepte, noch hat sich der Einzelhandel grundsätzlich mit der Frage der Raumplanung auseinandergesetzt. Dies gilt vor allem auf Bundes- und Kantonsebene. Ausnahmen wie z.B. das Versorgungskonzept für den Kanton Thurgau oder die Richtplanung «Einkaufszentren im Oberengadin» bestätigen die Regel.

Erstaunlicherweise gibt es auf Bundesebene (wie auch in den meisten Kantonen) nicht einmal Konzepte für die Zentrale-Ort-Struktur, die zumindest für die öffentlichen Dienstleistungen verbindlich wären. Auch in diesem Bereich herrscht der politisch motivierte Alltagspragmatismus gegenüber raumordnungspolitischen Grundsatzentscheidungen vor.

Von beiden Akteurgruppen werden sozusagen täglich eine Fülle von Entscheiden getroffen, die «einzelhandelswirksam» oder "raumwirksam» sind, ohne daß sie in weitergreifende Überlegungen eingebettet wären, geschweige denn koordiniert sind. Beispiele dazu sind:

1. Zonierungs- und Erschließungsentscheide, die große Einkaufszentren in neuen Gewerbezonen an verkehrsgünstigen Standorten ermöglichen, ohne daß der Einzelhandel ausdrücklich erwähnt ist. Das Baurecht unterscheidet nämlich in der Regel nicht zwischen Produktions- und Vertriebsunternehmungen.

2. Umweltschutzorientierte Verkehrsmaßnahmen in Stadtzentren, welche die Interessen des Einzelhandels nur indirekt berücksichtigen, da keinerlei Steuerungsmöglichkeiten bestehen. Allerdings zeigt das Beispiel der Fußgängerzonen, daß die Ergebnisse dieses pragmatischen Verfahrens beachtlich sind.

3. Unternehmerische Standortentscheide sowohl von Einzelgeschäften wie auch von Großverteilern, wie z. B. Betriebsschließungen, -erweiterungen, neue Geschäfte bzw. Filialen. Sie berücksichtigen ausschließlich betriebliche Gesichtspunkte, haben aber oft erhebliche Auswirkungen sowohl auf die allgemeine räumliche Entwicklung wie auch auf die Versorgungsstruktur.

Dies ist auch gar nicht anders möglich, da keinerlei Koordinations- und Ausgleichsmechanismen bestehen.

Zusammenfassend kann man sagen, daß ein solider ordnungspolitischer Konsens darüber besteht, daß sich der
Staat mit seiner Raumplanung nicht in den Einzelhandel einmischen sollte und der Detailhandel sich nicht verantwortlich zu fühlen braucht für seine Raumwirksamkeit. Oder: es ist allgemeine Politik, daß es keine «retail planning policy» gibt. Im anbrechenden Zeitalter der Deregulierung wird sich an diesem Zustand wohl auch kaum etwas ändern. Dank der nach wie vor steigenden Mobilität eines Großteils der maßgebenden Bevölkerungsteile besteht jedenfalls von der Nachfrageseite her kein Anlaß dazu; versorgungs- und sozialpolitische Motive (im Sinne einer "ausreichenden Versorgung») finden kaum ein Interesse. Dies ist allerdings aus umweltpolitischer Sicht wegen der damit generierten Zwangsmobilität nicht unproblematisch, wird sich aber erst ändern, wenn die Kostenwahrheit im Verkehrssektor stärker zum Tragen kommt bzw. andere stärkere Instrumente in der Raumplanung eingesetzt würden.

\section{Der Einzelhandel und seine Entwicklung}

\subsection{Zur Struktur des Einzelhandels}

Rund $40 \%$ des gesamten privaten Konsums der Schweiz fließen 1990 in die Kassen des Einzelhandels, auch Detailhandel genannt. Dieser Anteil hat in den vergangenen Jahren ständig abgenommen; 1970 lag er noch bei 53,3\% (RUDOLPH 1993/60).

1992 werden mit gut 60000 Verkaufsstellen die schweizerischen Detailhandelsumsätze von insgesamt 77,5 Mrd. Franken erwirtschaftet. Davon entfallen auf die Gruppe der Nahrungs- und Genußmittel 46,5\% und auf Nichtlebensmittel 53,5\% (HASEN/BIEL 1993/14). Das Verhältnis zwischen dem Food- und Non-Food-Bereich hat sich also weiter zuungunsten des Foodbereichs verschoben, wie Abb. 1 zeigt. 1989 war das Verhältnis noch halbhalb.

Martin Boesch, Prof., Dr.,

Susanne Schmid, dipl. Geographin,

FWR-HSG, Forschungsstelle für Wirtschaftsgeographie und Raumplanung, Postfach, 9011 St. Gallen. 


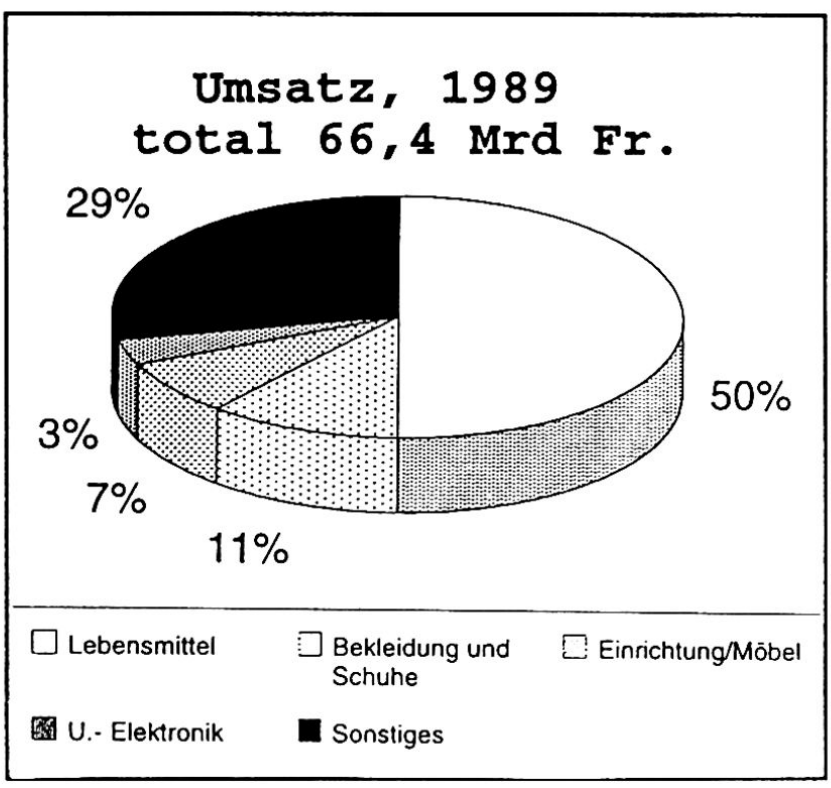

Quelle: WEINHOLD, H. (1991/41). Eigene Darstellung.

Die gesamte Verkaufffläche des Einzelhandels wird in der Schweiz 1990 auf über 7 Mio. Quadratmeter geschätzt, wobei die genaue Zahl mangels Angaben nicht erhoben werden kann. Dieser Wert setzt sich folgendermaßen zusammen (vgl. Abb. 2):

Abb. 2

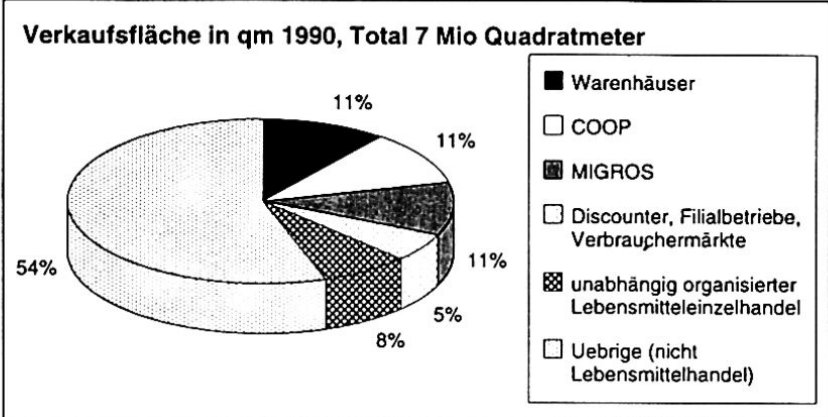

Quelle: HASEN/BIEL (1993/15). Eigene Darstellung

Rund je ein Zehntel der Verkaufsflächen werden von Migros, Coop und den Warenhäusern belegt, weitere gute $5 \%$ gehören der «dritten Kraft» (Usego/Waro, Pick \& Pay/ Distributa und Denner). Der unabhängig organisierte Einzelhandel belegt weitere $8 \%$ der Verkaufsfläche (vgl. Abb. 2).

\section{Marktanteile im Schweizer Detailhandel 1991}

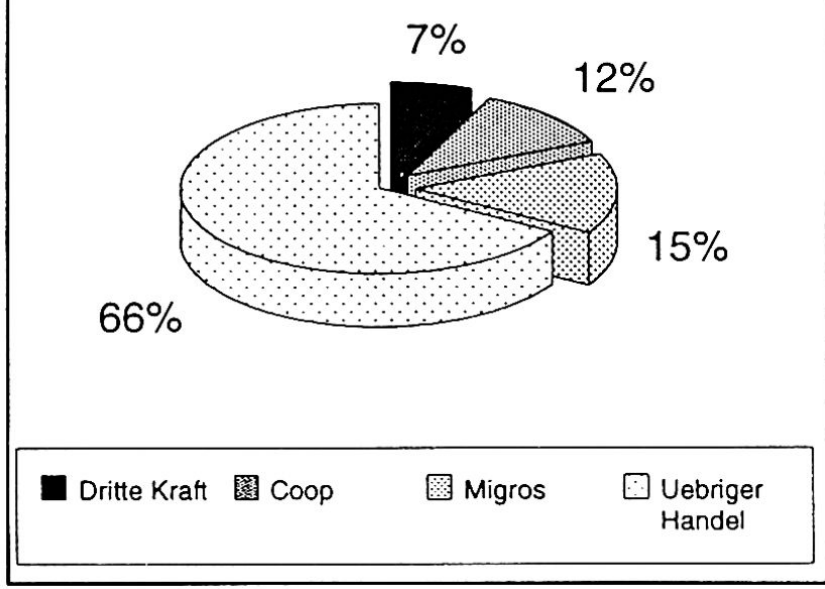

Quelle: HASEN/BIEL (1993/16). Eigene Darstellung.

Bezüglich des Marktanteils zeigt sich ein ganz ähnliches Bild, wenn auch hier die beiden genossenschaftlich organisierten Großverteiler Migros und Coop noch etwas stärker dominieren (vgl. Abb. 3).

Die beiden Großverteiler bewältigen 1991 zusammen $27 \%$ des gesamten Detailhandelsvolumens mit ihren $\mathrm{Ab}$ satzkanälen (HASEN/BIEL 1993/16). 1992 können sie diesen Anteil sogar nochmals auf ca. 30\% steigern (LÜTTGEN 1994/30). Entsprechend ihrer Entstehungsgeschichte sind die beiden Genossenschaften wesentlich stärker im Foodbereich tätig, wo sie zusammen $40 \%$ abzudecken vermögen. Die erwähnte "dritte Kraft» ist noch stärker im Foodsektor tätig (HASEN/BIEL 1993/16).

Zusammenfassend kann gesagt werden, daß im schweizerischen Einzelhandel der Lebensmittelhandel von zwei bis drei Marktführerinnen dominiert wird.

Da der Einzelhandel ein sehr personalintensiver Produktionszweig ist, verwundert es nicht, daß 1992 338000 Personen dort beschäftigt werden, was einem Anteil von rund $10 \%$ aller erwerbstätigen Personen in der Schweiz entspricht. Die Verteilung der Anzahl Beschäftigter nach den Kantonen bezogen auf 1000 Einwohner zeigt folgendes Bild (vgl. Abb. 4):

Der Kanton Basel-Stadt zeigt mit 67 Beschäftigten auf 1000 Einwohner $(=B / 1000$ E) die höchste Dichte, umgeben vom Kanton Basel-Land mit der geringen Dichte von nur gerade $35 \mathrm{~B} / 1000 \mathrm{E}$. Offensichtlich übernimmt die Stadt Basel für die Region die Versorgungsfunktion in einem hohen Maße. Die zweithöchste Dichte weist der Kanton Luzern auf, mit $56 \mathrm{~B} / 1000 \mathrm{E}$. Auch hier übernimmt die Stadt Luzern wahrscheinlich z. T. die Versorgungsfunktion der ländlichen Regionen der Kantone 


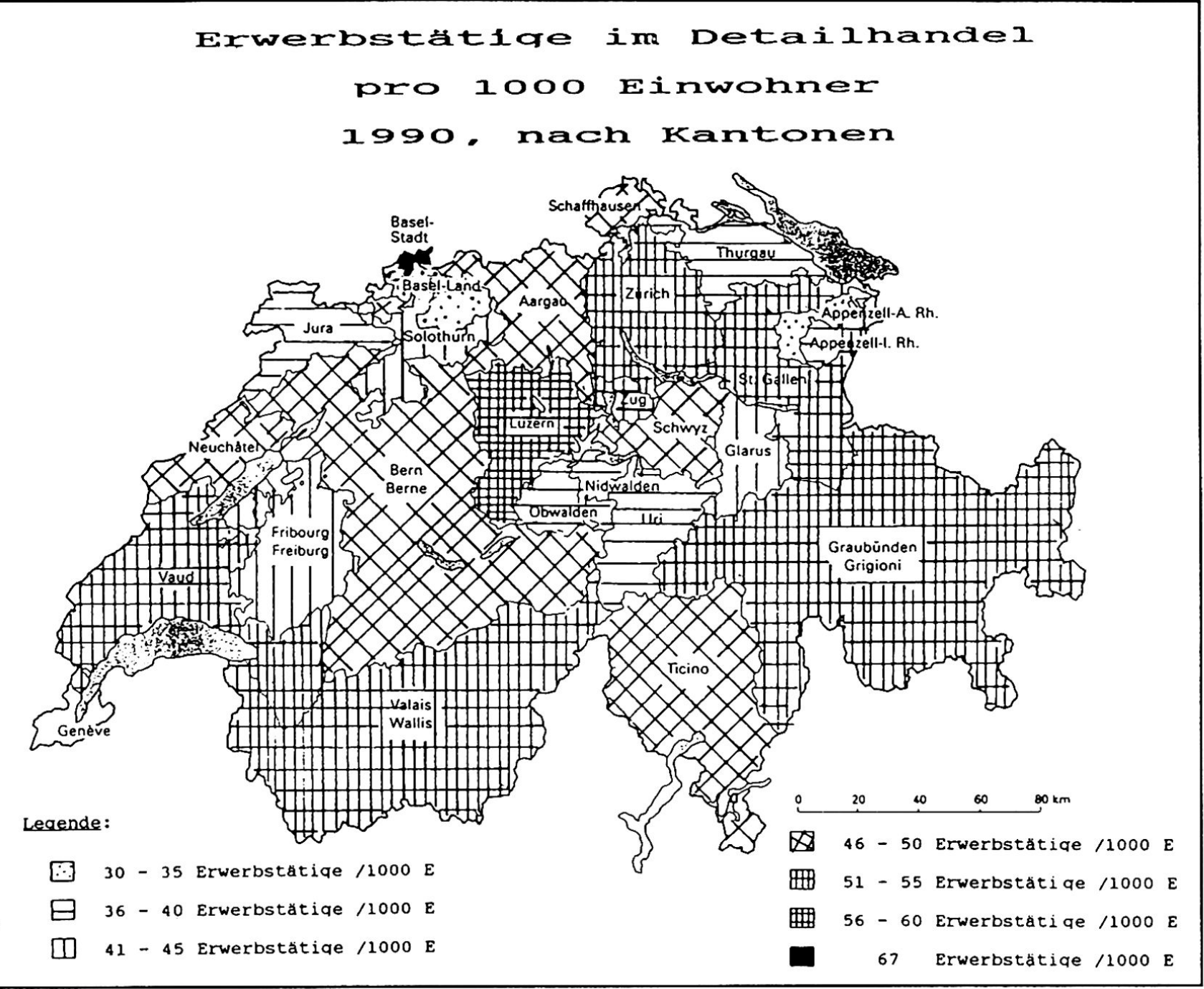

Quelle: Bundesamt für Statistik, Betriebszählung 1991. Eigene Berechnungen und Darstellung.

Uri, Obwalden und Nidwalden. Auch der Kanton Appenzell Außerrhoden mit der kleinsten Dichte von nur $30 \mathrm{~B} / 1000$ E wird offenbar stark von der Stadt St. Gallen mitversorgt. Daß die ländlichen Kantone Graubünden, Wallis und Waadt eine relativ hohe Dichte (50-55 B/1000 E) erreichen, kann mit der zerklüfteten Topographie erklärt werden. Zur minimalen Versorgung mit täglichen Gütern sind in diesen Gebieten relativ viele Verkaufsstellen nötig, wenn man davon ausgeht, daß die Wege zu den Läden nicht beliebig lang werden können. Unterscheidet man die erwerbstätigen Personen im Detailhandel nach deren Geschlecht, so fällt auf, daß die Mehrzahl Frauen sind. Der Anteil der Frauen beträgt durchschnittlich $62 \%$, erreicht aber im Kanton Uri den Höchstwert von $69 \%$. Am wenigsten Frauen im Detailhandel arbeiten im Kanton Genf, nämlich nur gerade $56 \%$.

\subsection{Entwicklungstendenzen im Einzelhandel}

Im Einklang mit anderen Wirtschaftsbranchen zeichnen sich im schweizerischen Einzelhandel Sättigungstendenzen ab. Das gesamtwirtschaftliche Wachstum ist im Jahr 1991 zum Erliegen gekommen. Die Detailhandelsumsätze sind 1992 und 1993 gegenüber dem Vorjahr real gesunken. Dabei muß beachtet werden, daß je nach Branchen die Verluste unterschiedlich ausfielen. Die Gruppe Nahrungs- und Genußmittel kann real bis 1992 noch Gewinne verzeichnen, erst 1993 muß sie sich mit dem Nullwachstum begnügen. Anders sieht das Bild bei den Bekleidungsartikeln und Textilwaren aus. Diese Gruppe erleidet bereits seit 1990 real Verluste (vgl. Abb. 5).

Der Konsument zeigt wieder ein höheres Preis-Leistungs-Bewußtsein, was sich auch im vermehrten Einkaufstourismus ins benachbarte Ausland zeigt: 1991 ge- 


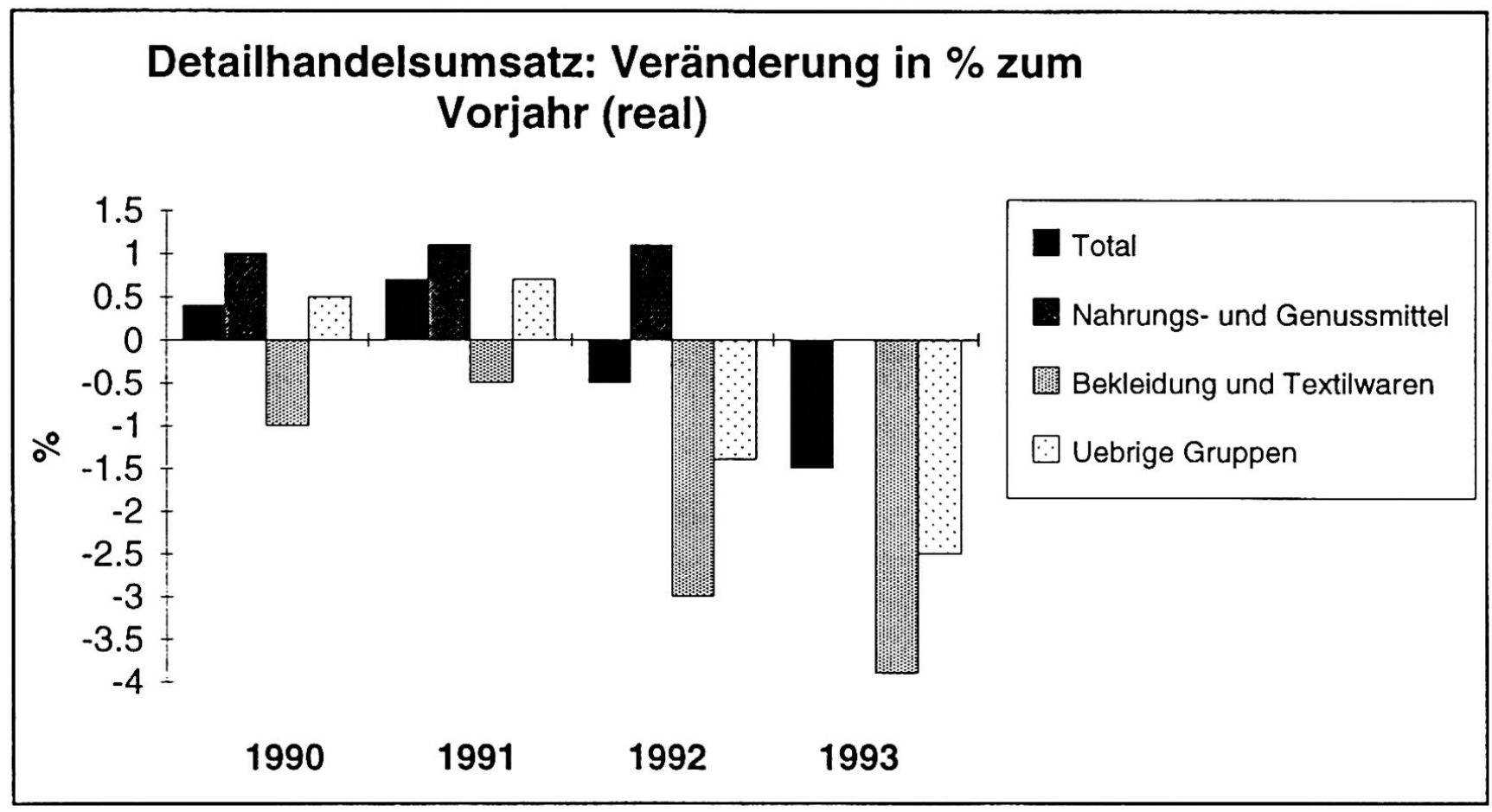

Quelle: Bundesamt für Statistik: Detailhandelsumsätze 1993. Eigene Darstellung.

hen dem Einzelhandel 900 Mio SFr. durch Kaufkraftabfluß in die grenznahen Gebiete verloren (RUDOLPH 1993/ $61)$.

Um die Verluste wieder wettzumachen, versuchen die schweizerischen Einzelhändler mittels Rationalisierungsmaßnahmen den Aufwand zu reduzieren, um so wieder mit Gewinn abschließen zu können. Die Optimierung der Logistik-, Lager- und Produktionskosten steht im Vordergrund.

Bezüglich der Anzahl der Verkaufsstellen liegen nur Zahlen aus dem Lebensmittelhandel vor, da dieser auch für die Versorgung mit lebenswichtigen Waren eine Vorrangstellung hat. Von 1985 bis 1991 nimmt die Anzahl der Verkaufsstellen im Lebensmittelhandel um $12 \% \mathrm{ab}$, gleichzeitig steigt aber die Verkaufsfläche um $14 \%$, der Gesamtumsatz um 33\% sowie der Umsatz je Verkaufspunkt um 50\% (vgl. Abb. 6).

Die Migros setzt z. B. 1993 77\% ihres Umsatzes in ihren 221 Einkaufszentren wie MMM-Zentren und MMMärkten um ( $=42 \%$ aller Verkaufsstellen). Mit dem Angebot der 304 kleinen Geschäfte wie M-Läden und S-Kleinläden $(=58 \%$ der Verkaufsstellen $)$ werden nur $23 \%$ des Umsatzes erzielt. Noch extremer zeigt sich dieses Bild, wenn man nur die größten Zentren (MMM-Zentren) betrachtet. Mit 38 Verkaufsstellen $(=7 \%)$ werden $28 \%$ des Umsatzes erwirtschaftet (MGB-Jahresbericht 1993).
Auch bei Coop Schweiz sieht die Situation sehr ähnlich aus: Mit den 43\% großen Verkaufsstellen werden auf einer Fläche von $61 \%$ der gesamten Verkaufsfläche $70 \%$ des Umsatzes erreicht. Die Hälfte aller Läden von Coop sind aber kleiner als $400 \mathrm{~m}^{2}$. Damit erzielt das Unternehmen aber nur gerade $17 \%$ des Gesamtumsatzes (Coop Schweiz, Geschäftsbericht 1993).

Abb. 6

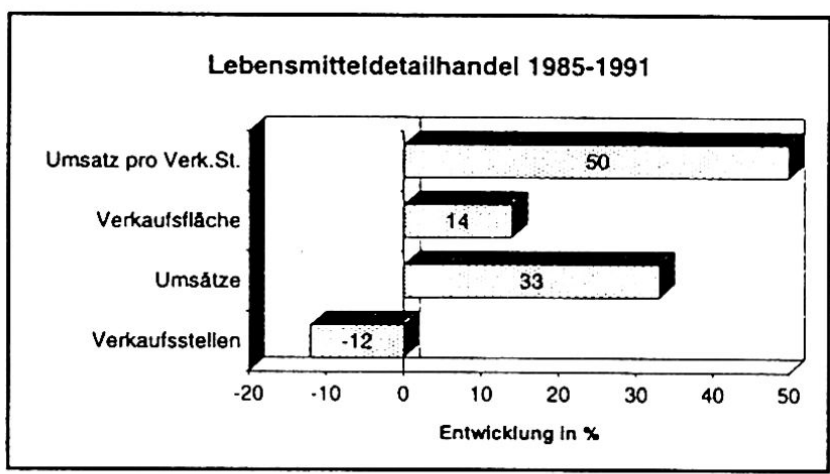

Quelle: HASEN/BIEL (1993/17). Eigene Darstellung. 
Hinter diesen Größen steckt die fast dramatische Entwicklung des Einzelhandels. Kleinere Geschäfte werden zugunsten von größeren Einheiten aufgegeben. Die groBen Einkaufszentren liegen mehrheitlich im gut erschlossenen Mittelland. Dies hat bereits zu Lücken im Verkaufsstellennetz geführt, die sich insbesondere in den Randregionen bemerkbar machen, während in den Ballungszentren von einem Überangebot an Verkaufsfläche gesprochen werden kann (sCHÜTZ 1992/19). Auch die häufig eingesetzten Verkaufswagen vermögen diese Lükke nur bedingt zu stopfen. Die Marktführerin Migros setzt z. B. 199288 Verkaufswagen zur Versorgung abgelegener Quartiere und Gemeinden ein, die insgesamt 3825 Haltestellen in 1426 Gemeinden bedienen, um so wenigstens eine minimale Versorgung $\mathrm{zu}$ gewährleisten (HASEN/BIEL 1993/17).

Auch die Coop Schweiz hat nach 1991 ihr Verkaufsstellennetz um weitere 300 Einheiten reduzieren müssen, da Verkaufsflächen unter $300 \mathrm{~m}^{2}$ nicht mehr in ihre Marktstrategie hineinpassen (SCHÜTZ 1992/20).

\section{Abb. 7}

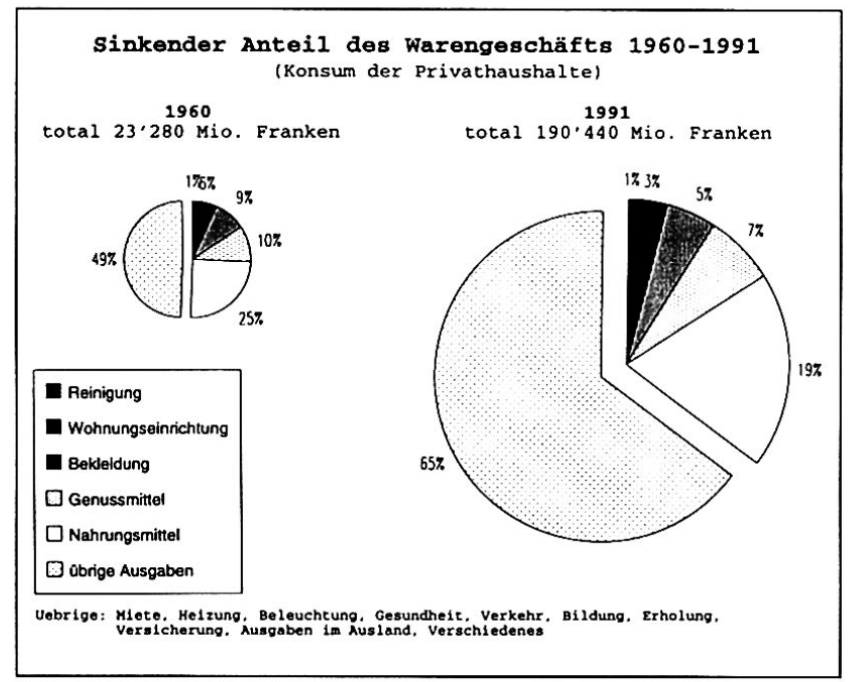

Quelle: HASEN /BIEL (1993/22). Eigene Darstellung.

Der in den letzten Jahren sinkende Anteil der Nahrungskosten am Gesamtbudget eines Privathaushaltes (vgl. Abb. 7), der sich zwischen 1961 und 1991 von 25\% auf $19 \%$ verringert hat, wird die Überlebenschancen kleiner Läden weiter verringern (HASEN/BIEL 1993/22).

\subsection{Einflüsse der (Stadt-)Entwicklung auf den Einzelhandel}

In Auswertung und Ergänzung zu den unter 2.2 aufgeführten sichtbaren Strukturveränderungen läßt sich festhalten, daß der Einzelhandel ein empfindlicher Gradmesser des allgemeinen Entwicklungstrends ist. Folgende Stichworte müssen hier genügen:

1. Wohlstandseffekte (verbunden allerdings mit einer Verschärfung der Verteilungsfrage) führen zu Verlage- rungen in "dynamische» Branchen, d.h. solche mit rasch wechselnden Modetrends und hohem Prestige (z. B. Sportartikel).

2. In Schweizer Städten kommt es kaum zu Slumbildungen; vielmehr halten die Verdrängungseffekte wirtschaftsstarker Nutzungen wie des Einzelhandels in den Zentren an. Sie werden allerdings durch einen Strauß von raumplanerischen Maßnahmen (u. a. Sondernutzungspläne) und eine zurückhaltende städtebauliche Politik gebremst.

3. Die wachsende, an leistungsfähige Infrastrukturen gebundene Mobilität bewirkt starke Verschiebungen der Standortqualität, ist doch die automobile Erreichbarkeit zu einer Schlüsselgröße geworden. Bevorzugte Stellen sind die Autobahnknoten, benachteiligte die Innenstädte. Es hat sich allerdings gezeigt, daß dieser Gradient durch den Einfluß anderer Standortqualitäten sowie die Probleme mit den Externalitäten des Verkehrs stark korrigiert wird. Insbesondere können ein gut ausgebautes Netz von öffentlichem Verkehr sowie ein dichtes urbanes Milieu eine ebenbürtige Erreichbarkeit bzw. Standortqualität generieren.

4. Der Rückzug aus der Gemeinschaft in die Privatsphäre und die verstärkte Akzentsetzung auf "wirtschaftliche Effizienz» führen zu mangelndem Verständnis für den Auftrag der Raumplanung: dem Ausgleich zwischen den Interessen und der Gestaltung eines hochwertigen Außenraumes.

5. Formen der sozialen Desintegration finden so leichter Nischen in den Innenstädten, wo sie manifest werden mangelnde Sicherheit ist die Folge. Sie schlägt durch auf das Ergebnis des Einzelhandels.

\subsection{Forderungen des Einzelhandels}

Das «Ladensterben» wie auch Verdrängungseffekte in Innenstädten haben sich still vollzogen: Anders als in der Landwirtschaft hat sich der organisierte Einzelhandel, die sogenannten "Gewerbekreise», nicht grundsätzlich gegen die Trends der Strukturveränderungen ausgesprochen, ja sie sind selbst ein starker Motor dieser Prozesse. Das zeugt von einem "gesunden» Wettbewerbsdenken, ohne Reflexion der Rahmenbedingungen noch der gesamtgesellschaftlichen Notwendigkeiten und der Folgen solcher Prozesse. Entsprechend kurz greifen die Forderungen des Einzelhandels an die Planungsbehörden: man will bloß "günstigere Rahmenbedingungen», d. h. maximale Erreichbarkeit für den MIV (motorisierter Individualverkehr), uneingeschränkte Nutzungsrechte, liberalisierte Betriebsbedingungen, günstige Steuerkonditionen und Sicherheit.

In der Tat sind dies die strategischen Erfolgspositionen der großen Einkaufszentren, gegen die die übrigen $\mathrm{Ge}$ schäfte bestehen müssen. Aus ressourcenökonomischer Sicht muß man feststellen, daß solche Kosteneinsparungen aus Externalitäten resultieren, die für die urbanen Kommunen nicht zugänglich sind bzw. sogar bewußt ab- 
gebaut werden sollten. Besonders typisch ist dies im Bereich der Bewirtschaftung des Privatverkehrs und bei der Sozialpflichtigkeit der Nutzungsrechte, also gerade bei den raumplanerisch besonders relevanten Kategorien. Aus diesen gegensätzlichen Sichtweisen ergab sich im Laufe der letzten Jahre eine starke Blockierung der Positionen, die den Eindruck erweckt, als ginge es tatsächlich um existentielle Fragen. Detaillierte Studien zeigen aber, daß der Einfluß der allgemeinen sozioökonomischen Rahmenbedingungen (insbesondere der Rezession) von viel größerer Tragweite ist. Der städtische Einzelhandel ist also in der Tat schlecht beraten, wenn er nicht versucht, aus der Kooperation mit den Gemeinwesen Verbesserungen seiner Position zu erzielen.

\section{Raumordnungspolitik}

Wie schon einleitend angedeutet, sind in der Schweiz die Planungsverfahren subsidiär organisiert. Bund und Kantone setzen nur die Rahmenbedingungen fest und delegieren die konkreten Gestaltungsmaßnahmen an die Gemeinden. Das Bundesgesetz über die Raumplanung (vom 22.6.1979) hält bezüglich der Versorgung in Art. 3 fest:

«Die Siedlungen sind nach den Bedürfnissen der Bevölkerung zu gestalten (...).

Insbesondere sollen...

...günstige Voraussetzungen für die Versorgung mit Gütern und Dienstleistungen sichergestellt sein.»

Dieser ziemlich unverbindliche Auftrag wird von den Kantonen und Gemeinden sehr zurückhaltend interpretiert: Abgesehen von Bestimmungen für Kern- oder Zentrumszonen, die ausdrücklich die Zulässigkeit von Geschäften, Handels- und Dienstleistungsbetrieben enthalten (vgl. z. B. PBG ZH §§ 50 und 51), gibt es kaum direkt detailhandelsbezogene Festsetzungen. Weder gibt es versorgungsorientierte Konzepte zur Siedlungsstruktur (auf regionaler oder städtischer Ebene) noch Instrumente zur ausdrücklichen Förderung bzw. Verhinderung von Geschäften, d. h. also keine konzeptionelle Standortpolitik für Versorgungsbetriebe.

Dazu kommt, daß infolge unseres föderalistischen Staatswesens die Kompetenzen im Planungs- und Umweltbereich auf viele lokale Behörden verteilt sind, was ein konsequentes Entscheiden der öffentlichen Hand erschwert und immer wieder «Schlupflöcher» öffnet, die die Externalisierung ermöglichen. Dies trifft vor allem in den großen Agglomerationen zu, bei denen Kernstädte und Vororte nicht die gleichen Interessen vertreten. Vor allem Großverteiler wissen dies zu nutzen.

Indirekt wirken sich allerdings vor allem zwei Instrumente auf die Geschäftsstruktur aus:

1. Die Erschließungs- und Umweltschutzvorschriften, die im Einzelfall dazu führen können, daß ein geplantes Einkaufszentrum nicht realisiert werden kann, weil es zuviel Emissionen (in Form von Kundenverkehr) generiert.
2. Die Wohnschutzvorschriften in Innenstädten, die im Einzelfall eine zu starke Expansion der Geschäftsnutzung einschränken könnten.

Der politische Prozeß zwischen Gewerbe und Lokalbehörden verläuft allerdings so, daß in solchen Fällen meist eine wirtschaftsfreundliche Lösung gefunden wird. Das Gewerbe hat nämlich eine sehr starke Position - sei es durch direkte Mandate in lokalen Behörden oder sei es durch informelle Einflußnahmen - und verschafft damit den wirtschaftlichen Argumenten ausreichende Akzep$\operatorname{tanz}$. Dies ist nicht weiter verwunderlich, wenn man berücksichtigt, daß die Lokalbehörden in der Schweiz in ihrer großen Mehrheit dem bürgerlichen Milieu zuzurechnen sind. In größeren Städten besteht ferner die Institution der Quartiervereine: dies sind meist gewerblich orientierte, privatrechtliche Vereine, die den wirtschaftlichen Interessen des Quartiers dienen und die bei Gelegenheit in quasi offizieller Funktion ihren Einfluß geltend machen. Mit zunehmenden Konflikten zwischen einer expansiven, wirtschaftlich orientierten Stadtentwicklungspolitik und den Anforderungen der Umweltund Wohnqualität im Laufe der 80er Jahre sind solche Einflüsse entscheidend geworden. Dabei wird oft übersehen, daß sich die Umweltschutzmaßnahmen sehr zum Wohl der Städte auswirken, was gerade dem Gewerbe wieder zugute kommt. Dennoch sind eigentliche Kooperations- und Mediationsmodelle bisher kaum zur Anwendung gekommen.

Diese Kommunikationsprobleme sind allerdings nicht außergewöhnlich, sondern geradezu typisch für den politischen Stil der 90er Jahre. Das politische System hat die zunehmende Komplexität der miteinander eng verflochtenen Sachfragen und die enorme Zunahme der Dynamik, was rasche und doch konsistente Entscheide erfordert, bisher nicht adäquat verkraftet. Während die 80er Jahre durch die beginnende Berücksichtigung der ökologischen Frage den notwendigen Ausgleich der öffentlichen Interessen brachten, blockieren heute Gewerbekreise den Dialog, wenn sie ihre Partikularinteressen nicht durchsetzen können. Dies ist insofern verständlich, als sie heute begründete Abstriche gegenüber der bisher üblichen, ja selbstverständlichen kostenlosen Beanspruchung öffentlicher Güter hinnehmen müssen - das Augenmaß für das Mögliche ist abhanden gekommen.

Demgegenüber spielen die KonsumentInnen und ihre Organisationen eine zwiespältige Rolle: sofern ökologische Kriterien zu einer Verteuerung der Produkte führen, ist die Unterstützung nicht sicher, was natürlich eine sehr kurzfristige Perspektive ist. Auch ist das individuelle Verhalten alles andere als konsequent: der große Wohlstand verleitet die KonsumentInnen zu erheblichen Zusatzkosten (höhere Wegkosten, Zeitkosten, unnötige Affektivkäufe), welche die Vorteile günstiger Angebote mehr als kompensieren. Zusammenfassend muß man feststellen, daß sich die Erwartungen in eine konsistente umfassende Raumordnungspolitik, die auch den tertiären Wirtschaftssektor umfaßt, nicht erfüllt haben und solche Ambitionen sich heute zunehmend als unrealistisch - wenn auch weiterhin als notwendig - erweisen. 


\section{Ausblick}

Geht man von der realpolitischen Situation aus und versucht daraus ein Zukunftsszenario abzuleiten, so kommt man unweigerlich zum Schluß, daß die Zeit der großen Konzepte und koordinierten Vorgehensweisen vorbei ist. Behörden aller Stufen wie auch Unternehmungen werden versuchen, möglichst ungebunden - sehr situativ jeweils «das Beste» zu machen - eine sehr ernüchternde, kurzsichtige Perspektive. Der Druck der Megatrends (Europäisierung und Ökologisierung) könnte allerdings dazu führen, daß allmählich ein größerer Handlungsbedarf erkannt wird. Wenn es größeren Bevölkerungskreisen (v. a. auch den zunehmend zahlreicheren Senioren) infolge ungenügender Mobilität nicht mehr gelingt, ihren Bedarf zu decken, oder wenn steigende Zwangsmobilität zu untragbaren Umweltbelastungen führt, wird raumstrukturell orientierte Versorgungspolitik (wieder) zu einem aktuellen Thema. Dann wird aber die Ausgangslage verändert sein: nicht mehr Erhaltung, sondern Beschaffung einer stärker dezentralisierten Versorgungsstruktur wird gefordert sein. Dazu fehlt aber der ordnungspolitische Rahmen völlig. Einzig die konsequente Umsetzung ökologischer Kriterien könnte dann als Korrektur wirken, während die sozialpolitischen Dimensionen nach wie vor ins Leere greifen. Ob dies dazu beitragen könnte, den lange fälligen «neuen Gesellschaftsvertrag» zu formulieren, erscheint aus heutiger Sicht allerdings mehr als fraglich. Das «Europa» der Wirtschaftsunion wird uns bei dieser Aufgabe jedenfalls kaum unterstützen.

\section{Literaturverzeichnis}

Bundesamt für Statistik (1993): Betriebszählung 1991. Gesamtdarstellung der Schweiz 1985/1991, Bd. 3 Erwerbsleben. Bundesamt für Statistik: Bern 1993.

Bundesamt für Statistik (1993): Betriebszählung 1991. Die Kantone im Überblick, Bd. 3 Erwerbsleben. Bundesamt für Statistik: Bern 1993.

Bundesamt für Statistik (1994): Detailhandelsumsätze Dezember 1993. Bundesamt für Statistik: Bern 1994.

Bundesamt für Statistik (1993): Statistisches Jahrbuch der Schweiz 1994. Neue Zürcher Zeitung: Zürich 1993.

Coop Schweiz: Geschäftsbericht 1993. Birkhäuser: Basel 1994.

DYCKERHOFF, S. (1993): Sortimentsgestaltung mit Dekkungsbeiträgen im Einzelhandel. Diss. HSG. Rosch-Buch: Hallstadt.

HASEN, H./BIEL, W. (1993): Perspektiven des Detailhandels. In: Die Volkswirtschaft 6/1993.

LÜTTGEN, F. (1994): Detailhandelsführer Schweiz 1993/ 1994. Handelskammer Deutschland-Schweiz, Zürich.

Migros-Genossenschafts-Bund Zürich (MGB) (1994): Jahresbericht 1993. Limmatdruck, Spreitenbach.

RUDOLPH, T. C. (1993): Positionierungs- und Profilierungsstrategien im Europäischen Einzelhandel. Diss. HSG. Thexis AG, St.Gallen

SCHMID, S. (1993): Auswirkungen der Fußgängerzone auf den Einzelhandel. (= FWR-Publikationen 24) FWR-HSG, St. Gallen.

SCHÜTZ, P.(1992):DerEinzelhandel vor EG '92. In: Die Volkswirtschaft 6/92.

WEINHOLD, H./BELZ, CH./RUDOLPH, T. (1991): Auswirkungen der Europäisierung auf den Einzelhandel in der Schweiz. Rüegger, Chur/Zürich. 